Universidad de Lima

Facultad de comunicación

Carrera de Comunicación

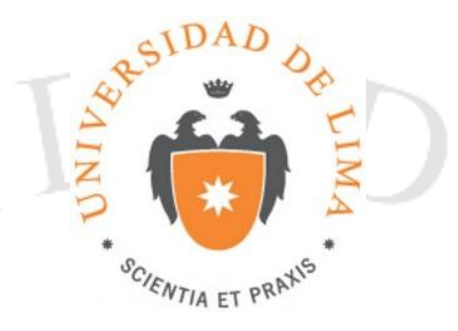

\title{
COMPONENTE COMUNICATIVO EN EL PROCESO DE INDUCCIÓN AL PERSONAL
}

Trabajo de investigación para optar la licenciatura en Comunicación

\section{Carla Avila Mendiolaza}

Código 20070949

\section{Asesor}

Lilian Kanashiro

\section{Lima - Perú}

Junio de 2016 


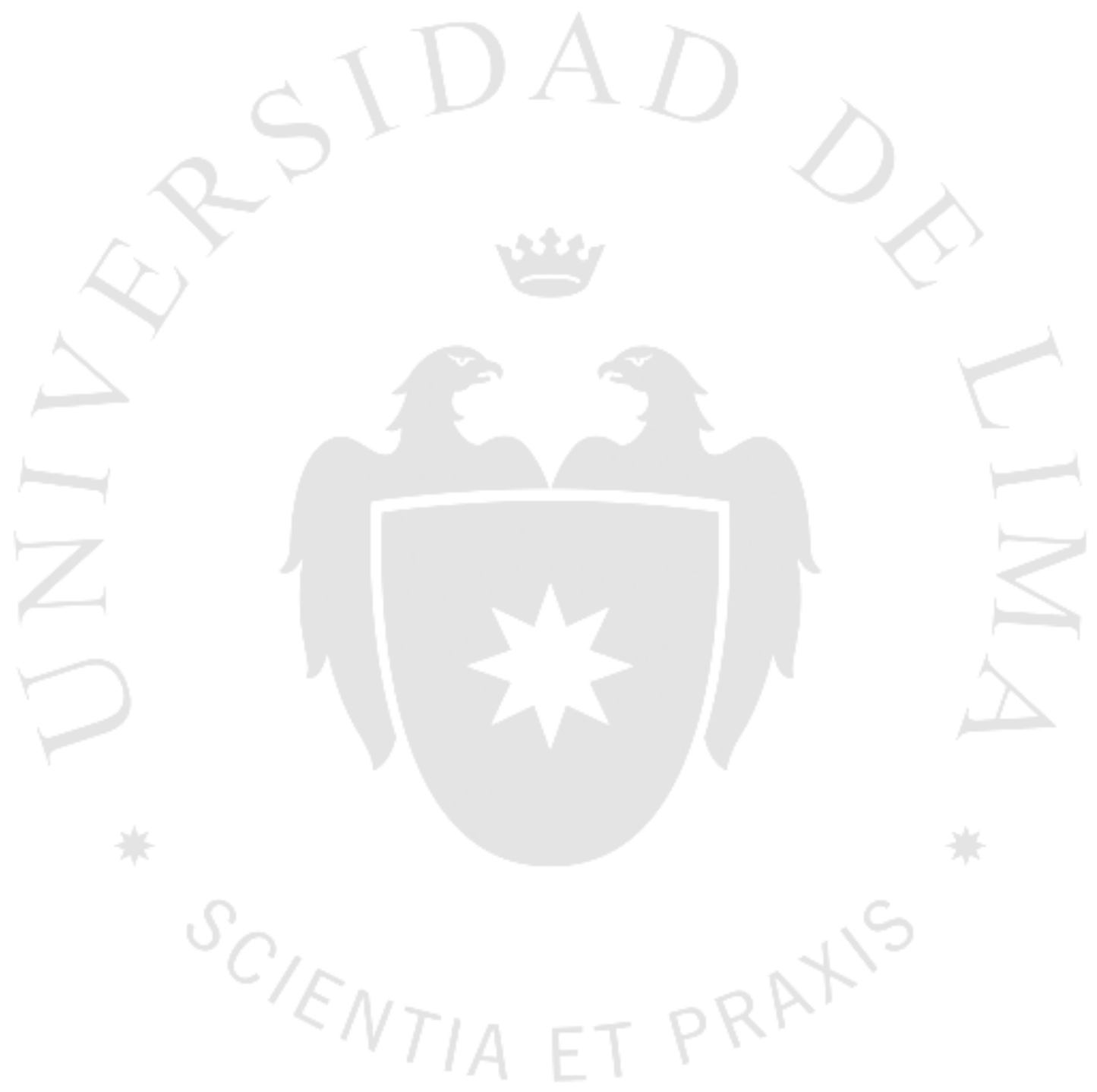




\section{COMPONENTE COMUNICATIVO EN EL PROCESO DE INDUCCIÓN AL PERSONAL}




\section{COMPONENTE COMUNICATIVO EN EL PROCESO DE INDUCCIÓN AL PERSONAL}

\section{Resumen}

La inducción al personal es un proceso en el cual la empresa brinda orientación que al nuevo colaborador sobre su puesto y funciones que desempeñará, incluyendo también un panorama general sobre la organización. Sin embargo, existe una tendencia a aprovechar este proceso para comprometerlo con la empresa, sus objetivos, y transmitir la cultura corporativa, además de la simplificación del contenido y los materiales entregados. Esto debido a que el contexto laboral actual comienza a exigir nuevas prácticas que fidelicen al colaborador desde el primer día de trabajo, es así que la comunicación torna un rol muy importante en cada parte del proceso buscando que los mensajes lleguen y sean interiorizados de la manera esperada. Este trabajo se llevó a cabo a partir de una investigación estrictamente cualitativa, en la cual se realizaron treinta y dos entrevistas a los encargados del proceso de inducción al personal de empresas del sector privado de distintos rubros llegando a encontrar que se está comenzando a incorporar a las charlas de inducción: talleres y detalles de bienvenida que involucran emocionalmente al nuevo ingreso, así como se está optando por reducir el material impreso, entregando la información en dispositivos de almacenamiento y plataformas digitales.

\section{Palabras clave}

Inducción al personal, comunicación, organización, medios, prácticas. 


\section{ÍNDICE}

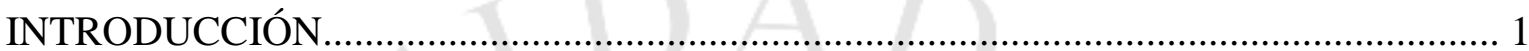

1. COMUNICACIÓN DESDE LA INDUCCIÓN …..........................................

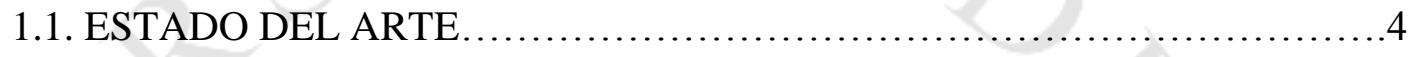

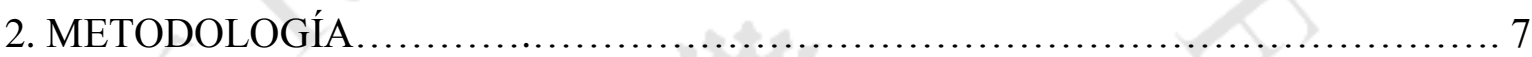

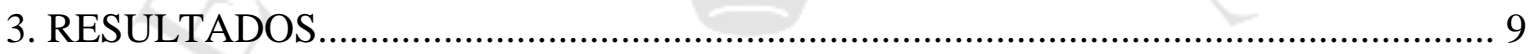

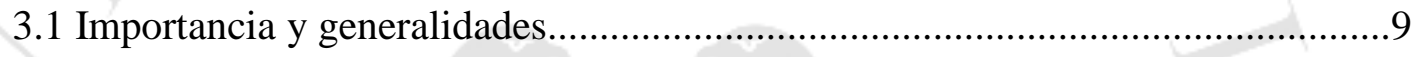

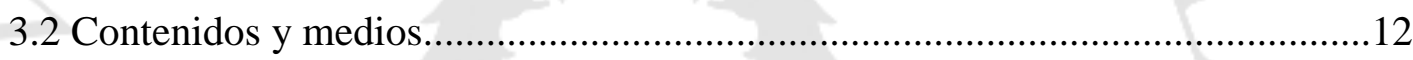

3.2.1 Contenido y tratamiento de la información en la inducción...............12

3.2.2 Medios............................................................. 15

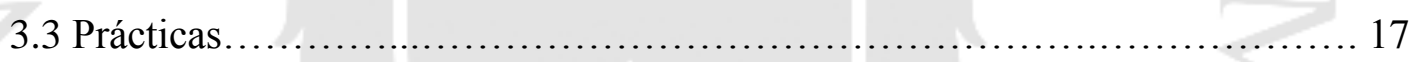

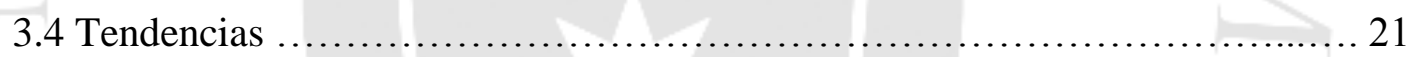

3.4.1 Acceso a la información y la tendencia a la digitalización del contenido ...................................................... 22

3.4.2 La cultura expresada desde la inducción ........................ 23

3.4.3 Proyectos a Implementar ....................................... 24

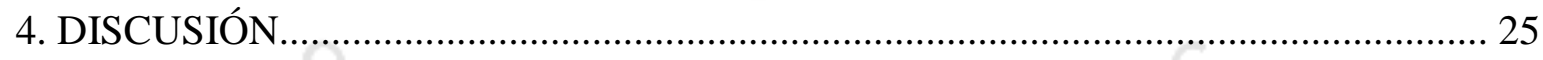

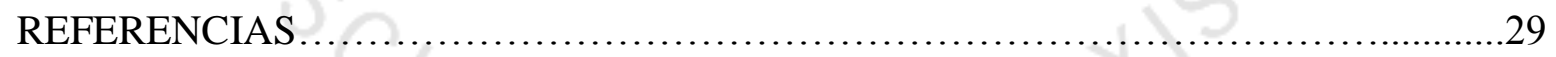

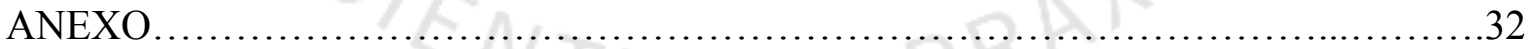




\section{INTRODUCCIÓN}

El proceso de inducción al personal es la orientación que brinda la empresa al nuevo colaborador sobre su puesto y las funciones que desempeñará, incluyendo también un panorama general sobre la organización. Si bien suele ser muy diferente en estructura, extensión y contenido dependiendo de las empresa que lo brinda ya sea por rubro, índice de rotación, entre otros factores, este suele ser la gran primera interacción formal de la organización con el nuevo empleado.

Sin embargo, el problema parece ser que este proceso está siendo subvalorado. Para empezar, no todas las empresas tienen un flujo o de manera estructurada lo que vendría a ser el proceso de inducción; además, no toman en cuenta que el primer contacto de los nuevos colaboradores con la organización puede ser un punto clave para iniciar una relación de compromiso con el trabajador: "Las primeras experiencias que vive un empleado en la organización van a matizar su rendimiento y su adaptación" (Grados 2003: $325)$.

Considerando que la adaptación es un proceso, la inducción sirve para ayudar a acelerarlo; por ello, el ingresar a un nuevo trabajo no solo significa aprender las nuevas labores, sino también a aprender la conducta común de los trabajadores, las convenciones no escritas, los comportamientos aceptables, la prácticas de la organización, entre otras cosas que, si bien se aprenden con el tiempo, pueden ser incluidas y facilitar la adaptación del nuevo colaborador. 
Además de ello, es tanta la información que reciben o que deberían recibir que, de no ser comunicada estratégicamente y tener un buen tratamiento además de un formato adecuado, terminará convirtiéndose en muchos manuales no leídos y olvidados en algún un cajón. El principal desacierto es que no se lleva un orden de los contenidos ni se consolida la información brindada por varias fuentes con el objetivo de que guarden relación y facilidad de comprensión. Los mensajes brindados — Según Calvo Pérez (2012) - deben ser sencillos y buscar trascender las capacitaciones siendo permanentes, repetitivos y consecuentes; ya que, de no ser así, podrían ser difíciles de recordar y por ende de aplicar.

Por ello, el presente proyecto de investigación tiene como finalidad sustentar que en un proceso de inducción el solo brindar información puntual sobre el puesto de trabajo y la historia de la empresa resulta ineficiente. La inducción se ha convertido en una herramienta de comunicación que puede ser utilizada para transmitir aspectos que facilitarán la adaptación de los nuevos trabajadores, como la cultura empresarial, los objetivos de la organización y cómo su labor se relaciona con ellos; así como también puede llegar a generar bienestar y compromiso con la empresa desde el primer día.

Por otro lado, también se tomará en cuenta el componente principal de la inducción, que es la manera en la que se brinda la información al nuevo colaborador, los mensajes considerados como indispensables, los medios en los que se transmiten y la variedad de materiales y actividades que se dan a lo largo del proceso. Nuestro objetivo general, entonces, será evaluar el componente comunicativo en el proceso de inducción al personal en empresas del sector privado, buscando tener una visión general del rol de la comunicación en este proceso, su estado actual y las tendencias que comienzan a manifestarse.

Además, se busca identificar los medios o materiales de comunicación, así como sus respectivos contenidos para ver si guardan relación, un formato común y si el tratamiento que le dan a la información responde a la necesidad y el contexto en los cuales se muestra; finalmente, buscaremos conocer las prácticas realizadas dentro de este proceso, cómo impacta en la adaptación del nuevo colaborador al puesto y su relación con su nuevo empleador. 


\section{COMUNICAR DESDE LA INDUCCIÓN}

La inducción al personal — según Valera y Dessler (2011) - es la etapa en la cual se brinda a las nuevas contrataciones la información básica que necesitan para realizar sus labores de forma satisfactoria, además de ser un elemento del proceso de socialización del trabajador nuevo que tiene que realizar el empleador. Por ello es importante que inicie desde el primer momento en el que los nuevos colaboradores pasan a ser miembros oficiales de la empresa.

Este proceso - según Gómez, Balkin y Cardy (2008) - se divide en dos grandes partes: la orientación, que consiste en brindar la información básica sobre la empresa y el puesto; y la socialización, que cuenta a su vez con tres partes: la previa, de encuentro y de adaptación. La etapa previa consta de las expectativas y el conocimiento que se tiene de la empresa por el boca a boca; cuando esas expectativas no se llegan a cumplir, puede provocar mal rendimiento o alta rotación. La etapa de encuentro es el inicio de la vida laboral dentro de la empresa, donde el colaborador necesita información sobre las políticas, procedimientos, relaciones jerárquicas, etc. Finalmente, la etapa de adaptación es aquella donde los trabajadores se empiezan a sentir parte de la empresa, dando como resultado que se sientan bien en su puesto y roll en la empresa.

Para que cada una de esas etapas pueda llevarse a cabo y tener una inducción de éxito, existe un elemento fundamental, la comunicación. Esta no solo sirve para dar instrucciones textuales y coordinar reuniones por correo electrónico dentro de una organización, sino que "Es la manera de relacionarse con otras personas a través de ideas, hechos y valores. La comunicación es el proceso que une a las personas para compartir 
sentimientos y conocimientos y que comprende transacciones entre ellas" (Chiavenato 2011:50)

Por eso su rol es tan importante dentro de este proceso que ha ido cambiando y estructurándose en los últimos años, ya que al ser dirigido a personas que inician una nueva etapa y se encuentran con muchas inquietudes y expectativas, hoy en día no basta con darles un cuadernillo con la historia de la empresa, si no que puede ser el momento para utilizar de manera estratégica la comunicación para anticiparse a los cuestionamientos, motivarlos y que vean a su nuevo centro de trabajo justo como este quiere ser visto o reconocido.

Si se logra comunicar adecuadamente la información necesaria y generar un ambiente positivo de bienvenida, la inducción suele generar varios beneficios para la empresa — según Jaime Grados (2003) —, como reducir costos en reclutamiento y selección, crear una actitud favorable hacia la empresa, acelerar la integración al ambiente laboral, integrar los objetivos de la organización con los de la persona, además de permitir al individuo realizar sus nuevas labores con facilidad, ahorrar tiempo para lograr estándares de calidad, incrementar el porcentaje de aceptación de las normas, entre otras mejoras. En general, aplicar este proceso ayuda a reducir el tiempo de adaptación y a crear no un primer encuentro, si no, el inicio de una relación entre ambas partes.

Pero más allá de su estructura y sus beneficios, Dessler (2009) manifiesta que la inducción al personal debe cumplir como mínimo con cuatro objetivos: primero, que el nuevo empleado se sienta bienvenido, que vea que lo estaban esperando; segundo, tiene que adquirir una comprensión general sobre la organización, que incluya su misión y visión; tercero, debe ayudar a que quede claro lo que se espera de su trabajo y su conducta; y, por último, iniciar el proceso de conocer la manera en que la empresa actúa y hace las cosas.

\subsection{Estado del arte}


Anteriormente se han realizado investigaciones y proyectos respecto al proceso de inducción al personal, siendo la mayoría casos de estudio o proyectos de implementación y estructuración del proceso en una sola organización. Si bien no se toca muchos aspectos sobre el componente comunicativo, llegan a conclusiones que podrían respaldar el presente trabajo.

En el caso de los trabajos revisados que giran en torno a empresas que no tienen este proceso institucionalizado, llegaron a las siguientes aproximaciones. Primero, solían ser empresas donde los colaboradores no se sentían valorados y donde en un inicio no sabían cómo desenvolverse ni realizar sus funciones, encontrándose también problemas de identificación con la organización; segundo, terminaban considerando indispensable que se dé el proceso en toda organización porque mejora el desempeño y adaptación de los colaboradores, como mencionan García y Oliveiro (2014), además de recomendar que este debe fusionar lo teórico y lo práctico (Jaramillo y Díaz 2011); y tercero, que hasta cierto punto la inducción debe buscar un involucramiento más allá de lo laboral con el nuevo personal (Jofre 2007), ya que tiene que haber una coincidencia de los valores de la compañía y del individuo.

Por ello, al final siempre suelen sugieren poner en práctica e institucionalizar el proceso para que los nuevos trabajadores sean capaces de reconocer los objetivos de su labor, conocer más la institución y lograr el empoderamiento de su puesto de trabajo (Paillacho Anchapaxi 2011) Además, se considera la mejor oportunidad para dar a conocer los objetivos de la empresa, permitiendo que realicen su trabajo teniendo una meta (Arellano Reinoso 2014) y mejorando así su eficacia y compromiso.

Por otro lado, los trabajos que buscaban evaluar procesos de inducción ya implementados, resuelven que la mayoría carece de dos partes que consideran muy importantes: el seguimiento y la retroalimentación de la inducción que sirven - como menciona Mendoza Jiménez (2013) — para identificar los puntos claves (lo que no quedó claro para el trabajador), con el fin de reforzarlo o tomar acciones concretas sobre los mismos; además, no realizar el seguimiento podría ser causa del trabajo en forma mecánica y errores en el proceso de adaptación. 
Cabe resaltar que varios mencionan la figura de un mentor, ya que es bueno que los nuevos ingresos sientan la libertad de acudir a una persona con experiencia, que conozca las labores y la empresa, que pueda resolver sus dudas restantes. Además, algunos llegaron a la conclusión de que este proceso involucra un presupuesto que puede convertirse en una inversión a largo plazo — como menciona García (1999)—, que llega a ser rentable debido a que desde el inicio produce profesionales más capacitados y ayuda a reducir la pronta deserción.

Los autores Cárdenas, Soriano Muñío, Calvo y Oliva (2009) presentaron una lista de objetivos que consolidan de buena manera los presentados por otras investigaciones y que inclusive podrían dar pie a indicadores para respaldar la rentabilidad: disminución del lead time hasta "empleado productivo", disminución de rotación, mayor satisfacción e integración empleado, mejora de la imagen de empresa como employer of choice, incorporación de mejores prácticas basadas en la experiencia de nuevo empleado, optimización del proceso de selección y, finalmente, asimilación de valores y cultura de la empresa.

En un caso más relacionado con esta investigación, haré mención de una tesis realizada por Rocío Huerta en el año 1991, titulada La comunicación organizacional, los programas de inducción al personal y su importancia en la dinámica de la organización. En primer lugar, la autora deja en claro que este proceso está íntimamente ligado a la comunicación al decir que la inducción es parte de una política concreta de comunicación ya que busca a través de ella que los nuevos se acoplen al sistema organizacional. Incluso sugiere que este proceso sea responsabilidad de un comunicador organizacional debido a que es la persona óptima para tratar correctamente el contenido y presentarlo de la mejor manera bajo los medios adecuados. 


\section{METODOLOGÍA}

Esta ha sido una investigación cualitativa debido a que ha sido enfocada solo desde la perspectiva del empleador, donde se busca ahondar en el proceso de inducción para encontrar, además de datos puntuales sobre estructura y objetivos de este, motivos, experiencias, grados de relevancia de sus componentes, nuevas tendencias, etc.

El muestreo fue por juicio. Según Julio Mejía (2000), este tipo de muestreo consiste en la selección de las unidades solo a partir de criterios conceptuales, siendo las variables que definen la composición de la muestra definidas por el investigador. En el caso de esta investigación, la población estudiada está compuesta por las personas encargadas del proceso de inducción al personal independientemente del puesto que tengan; es decir que, si el proceso está bajo la responsabilidad del área de Recursos Humanos, no se buscó entrevistar al gerente del área necesariamente, sino al subgerente, analista o cualquiera sea la posición de la persona que coordina directamente y/o lleva a cabo la inducción. Esto con el objetivo de conocer íntimamente el proceso, detalles y percepciones de quienes lo realizan de manera continúa bajo lineamientos de la organización y sus diversos puntos de vista y consideraciones de lo que se debería o no hacer, además de lo que se toma en cuenta durante el desarrollo de este.

Para el desarrollo de esta investigación, se realizó treinta y dos entrevistas a profundidad a personas del perfil anteriormente mencionado, en distintas empresas del sector privado, abarcando diferentes rubros. Se realizó esa cantidad de entrevistas debido a que es la cantidad en la cual se sugiere (y se alcanzó) el punto de saturación. Mejía (2000) 
sostiene que, de veintiséis a treinta casos, se redunda en el conocimiento de las pautas de los procesos sociales. Así es como la validez de la generalización de la muestra es resultado del conjunto de casos "tomados del mismo conjunto de relaciones socio estructurales, que se apoyan unas a otras y construyen todas juntas un cuerpo sólido de evidencias" (Bertaux y Bertaux-Wiame 1993, citado en Mejía 2000: 172).

Las entrevistas se realizaron entre los meses de abril, mayo y junio del año 2015, ubicando a los puestos que calzaran con el perfil mediante contacto a colaboradores que trabajaran en las diferentes organizaciones, por referidos de las personas que iban siendo entrevistadas, además de contactos buscados por las páginas web de las empresas y por redes sociales, haciendo el primer contacto usualemente vía correo electrónico. 


\section{RESULTADOS}

La investigación está enfocada en el proceso de inducción a la empresa, mas no profundizará en la inducción al puesto. Para mayor organización de los hallazgos, han sido distribuidos en: importancia y generalidades; contenidos y medios; prácticas; y, finalmente, tendencias.

\subsection{Importancia y generalidades}

La estructura del proceso de inducción al personal varía en las organizaciones por diversos factores: fecha de ingreso, cantidad de colaboradores, rubro, entre otros; sin embargo, suelen coincidir en una estructura básica: presentación de la empresa, información sobre condiciones laborales como detalles del contrato, beneficios y una inducción al puesto que por lo general depende del jefe directo.

Hay un aspecto importante que resaltar antes de continuar con los demás hallazgos y es que la tercera parte de las organizaciones que participaron de la investigación recién han iniciado el proceso de inducción hace un año o menos y que varias de las otras empresas restantes han restructurado el proceso o se encuentran en proyectos para mejorarlo actualmente:

Yo llegué a la compañía hace dos años y cuando llegue no había inducción, era la típica, llenabas tus documentos en el sistema, ibas a tu sitio y te decían lo que tenías 
que hacer, no conocías nada de la empresa, ni la estructura, objetivos, nada [...] y lo más importante era que sepan quiénes somos $[\ldots]^{1}$.

Esta situación nos indica que el contar con una inducción al personal está cobrando mayor importancia para las empresas. Se puede dar por cambio de dueños, objetivos o visión, como en el caso de Frecuencia Latina cuando pasó a ser Latina, donde se está iniciando un proceso de inducción antes inexistente; además de otros motivos, como utilizarlo para contrarrestar un tema de falta de identificación con la empresa.

Por otro lado, también consideran que ha crecido su importancia debido a la coyuntura actual. Helena Pinilla, Mónica Pardo y Vania Dazza (2014) mencionan, en un informe compilado por Justo Villafañe, dos puntos resaltantes del CADE 2013 (Encuentro anual de empresarios-Perú) El primero, que el stake holder más crítico hoy para las empresas peruanas no era el cliente si no el colaborador y el segundo que los dos grupos económicos más grandes, en Perú, tenían en sus pilares estratégicos, para el siguiente año, la generación de compromiso al interior de sus organizaciones. "El tema de la guerra de talentos es tan fuerte que ya el nivel remunerativo no te va a jalar una vacante, entonces tienes que darle valor agregado al trabajo desde el inicio, si no ten por seguro que todo lo que enseñes se lo lleva otro",2.

Sin dejar de mencionar que tiene un impacto en lo económico, una persona encargada del proceso mencionó que la rentabilidad era vital: si alguna persona se iba en un periodo corto de tiempo, implicaba un impacto económico; por lo cual, como parte de su labor, tenían que "enamorarlos", lograr que se "enganchen" con la empresa desde el primer día, al igual que otras empresas como Grupo Siglo, quienes comentaron que para ellos el tiempo valía oro, pero que la inducción más que un gasto era una inversión.

Por lo general, es el área de Recursos Humanos la responsable del proceso porque la información que se brinda es casi íntegramente la que ellos manejan y también por tener cercanía al nuevo ingreso. Una experiencia resaltante es la de Deloitte, que hasta hace poco tenía como responsable al área de Capacitación y que actualmente fue derivado a Recursos

\footnotetext{
${ }^{1}$ Comunicación personal con Christian Chapman (subgerente de Desarrollo de Talento, Clima y Cultura en BNP Paribas Cardif), el día 12 de junio de 2015.

${ }^{2}$ Comunicación personal con Vanessa Castillo (HR Business Partner Supervisor en British American Tobacco), el día 10 de junio de 2015.
} 
Humanos; ya que se dieron cuenta que, durante las inducciones, los ingresos aparentaban entender todo y, cuando aparecía el reclutador para exponer, lo llenaban de preguntas debido a que le tenían más confianza. Ahora, con el cambio, manifiestan que están alcanzando su objetivo de que la inducción sea una charla u etapa en la que los nuevos ingresos resuelvan todas sus dudas.

Las empresas coinciden en que el objetivo del proceso radica en brindarle la información necesaria para su desenvolvimiento laboral y una rápida adaptación:

He estado en otras empresas donde la inducción no es tan fuerte y se ve una diferencia, hay vacíos de información, de enganche y el mismo colaborador tiene que proveerse de ella y no siempre de una fuente formal y una empresa es responsable de darle la información y herramientas, y la misma información es la herramienta que el nuevo colaborador necesita... yo te voy a exigir productividad, pero si no te doy el conocimiento inicial ni de tu equipo u objetivos, de dónde vas a trabajar, ¿Cómo?³

Además, para algunas lo más importante es que se impliquen con los objetivos de la organización y los tomen como suyos al momento de trabajar: "si una persona no conoce los objetivos de la empresa, solo viene a trabajar por trabajar pero no tiene idea para qué, nosotros queremos que tengan un motivo más allá del salario" ${ }^{\text {. }}$

Se considera de suma importancia que casi la totalidad de las empresas crean que se puede comenzar a comprometer y fidelizar al nuevo colaborador desde el momento de la inducción, si bien varias manifestaron que a sus programas aún les falta para lograrlo. La mayoría considera que el componente motivacional es fundamental debido a que puede afectar en cierta medida el desempeño del nuevo colaborador y que inclusive la actitud hacia la empresa podría ser positiva desde el inicio por un tema de agradecimiento hacia una bienvenida cálida.

Solo seis empresas manejaban la inducción de manera individualizada; es decir que la gran mayoría de inducciones se dan de manera grupal. La mayor diferencia estaba en su extensión (que depende de la cantidad de información y aspectos normativos) y el

\footnotetext{
${ }^{3}$ Comunicación personal con Lorena García (especialista corporativo en Selección en Casa Andina), el día 25 de junio de 2015.

${ }^{4}$ Comunicación personal con David Carpio (analista de Desarrollo Organizacional en Imedia Acceso Group), el día 26 de junio de 2015.
} 
momento en que se dan. En cuanto al inicio, las empresas que tienen fechas fijas de ingreso de personal, suelen hacer la inducción el primer día y, las que no, una vez al mes o cada dos meses; pocas son las que tienen implementado el proceso y que dejan pasar más tiempo del mencionado.

Cabe resalar finalmente que, como consecuencia de que sea un tema relativamente nuevo la implementación de este proceso de manera estructurada, que demanda más tiempo, logística, etc., varias empresas (incluyendo en menor medida alguna que ya lo tiene implementado hace un par de años) manifestaron la molestia de que algunos mandos medios o jefaturas no vean la importancia que ellos consideran que tiene el que la inducción se dé desde un inicio:

Creo que igual con todo hay jefes que te dicen que quieren ya al nuevo ingreso, que no importa, que después se les programe la inducción, pero al final si es que se da el caso están preguntando y porque el aún no tiene correo, uy se han demorado mucho, porque no sabía de esto y solo refuerzan el hecho de que ese proceso es muy importante ${ }^{5}$.

\subsection{Contenidos y medios}

\subsubsection{Contenido y tratamiento de la información en la inducción}

De manera universal, todas las empresas incluyen temas básicos de Recursos Humanos; entre ellos, detalles de la condición del contrato, gestión de vacaciones, póliza de seguro, planilla, línea de carrera, capacitaciones (si las tuvieran) y presentación del personal del área para que sepan con quien pueden hablar de temas más específicos, esto seguido por una presentación formal de la empresa, a qué se dedica, su historia, su misión, visión y valores.

${ }^{5}$ Comunicación personal con Paola Carozzo (subgerente de Áreas de Apoyo, Recursos Humanos, en BBVA), el día 19 de mayo de 2015. 
Además, la mayoría de empresas cuenta con una segunda parte que incluye la presentación de las todas las áreas de la empresa (al menos de las más importantes) y, en caso de ser inducción individualizada, de las áreas que tienen relación directa con el puesto. Las empresas que tienen ciertas regulaciones y obligaciones por ley (como las financieras o mineras) incluyen temas como seguridad y salud en el trabajo, políticas ambientales, entre otros, y suelen ser más estructuradas. Por ejemplo, en Volcan, la inducción general en Lima dura dos días; sin embargo, la inducción para el personal que trabajará en sedes de provincia y directamente en la mina tiene una extensión de seis días de acuerdo a lo que indica la ley.

Otros contenidos entregados a los nuevos colaboradores a manera de información son una diversidad de manuales y políticas que se manejan de manera interna, que se caracterizan por tener una gran extensión y suelen ir desde la malla de capacitación, flujos y políticas de acción, manual de marca, reglamento interno de trabajo, hasta manuales de prevención de lavado de activos, dependiendo del rubro de la organización. Además, cerca de la cuarta parte de las empresas suele mencionar cómo se da el flujo de la información en la organización y qué medios utilizar para procedimientos claves y/o su desenvolvimiento diario.

Se suele dar a conocer también aspectos propios de la filosofía de la empresa, si es que pertenecen a una corporación, como en el caso de Chillis: "Como te comentaba nos regimos por estándares que necesitan conocer para cumplirlos y además nosotros tenemos nuestra "Pasión" que es que la gente esté contenta, tanto de afuera como de dentro [...] desde la inducción te incrustan el chip de hacer tu trabajo divertido pero con responsabilidad", incluso algunas que incluyen contenido acerca de la cultura de la empresa, tema que se profundizará más adelante.

Para terminar de hablar del contenido, que como vimos es muy diverso en el proceso dependiendo de la empresa, es importante considerar la creación y consolidación de este. Si bien el proceso y su contenido en general suele ser responsabilidad del área de

\footnotetext{
${ }^{6}$ Comunicación personal con Ángel Loyola (entrenador en Chillis), el día 30 de junio de 2015.
} 
Recursos Humanos, esta suele tener aliados estratégicos en otras áreas que dan información y soporte.

Pocas empresas contaban con un área de comunicación y menos trabajaron las presentaciones y materiales de la inducción con ella. Sin embargo, se registraron casos donde esta sirvió de soporte, como en Deprodeca, que cuenta con proceso de inducción desde este año, el cual incluye tres videos y una presentación bastante gráfica realizada entre Recursos Humanos y Comunicaciones; inclusive, el soporte de esta área no solo se da en la elaboración de contenidos y formato, sino, como en el caso de Natura, asesorando en varios aspectos hasta en la capacitación de los facilitadores. Esta empresa tiene instituida una inducción de dos semanas replicada a nivel transnacional; pero recién este año optó por mejorarla buscando soporte en su área de Comunicaciones, aumentando así el índice de satisfacción de los nuevos con el proceso:

El apoyo de comunicación interna es primordial... les hemos dado orientaciones de como exponer efectivamente, comunicación asertiva, como transmitir el lenguaje de marca, todos los facilitadores los han recibido y ya están capacitados, alineados con este mismo estilo, lenguaje, forma y esencia de comunicación ${ }^{7}$.

A pesar de no contar con un área de Comunicaciones, muchas empresas consideran importante que todo el contenido brindado sea juntado y revisado por una sola área o persona; ya que, al ser mucha la información, si no se comunica adecuadamente, puede no ser asimilada de la manera esperada. Por ejemplo, en Saga Falabella, el área de Desarrollo y Aprendizaje consolida la información, le da un solo formato y busca hacer más dinámica la presentación.

Sin embargo, aún existen empresas que lo manejan de manera distinta, como una minera donde cada área es responsable de lo que presenta ante los nuevos ingresos e incluso, hasta hace muy poco, no tenía siquiera el mismo formato para las diapositivas. Algunas empresas comentaron que, con ese tipo de esquema, muchas veces no se tiene un resultado positivo: "se revisó todo el material para verificar y asegurar que no se repita la

\footnotetext{
${ }^{7}$ Comunicación personal con Rodrigo Chávarri (analista de Comunicación Interna en Natura), el día 17 de julio de 2015.
} 
misma información, que todo esté alineado con los valores, que vayamos en la misma línea de comunicación, que no haya nada que se contradiga como sucedía antes"8.

\subsubsection{Medios}

Tanto para las inducciones grupales como para las individualizadas, se utiliza, de manera universal, presentaciones en formato Power Point, debido a que permite plasmar en las diapositivas la estructura de la presentación y ser un soporte visual. La tendencia es comenzar a incluir plantillas que permiten que los diferentes temas y exposiciones guarden una línea gráfica y sensación de continuidad.

Se encontró también que se recurre de manera regular al uso de videos. El más utilizado es el video institucional, que suele mostrar de forma inspiradora la labor de la empresa, historia, número de colaboradores, sedes, posicionamiento, entre otros $\mathrm{y}$, en algunos casos, incluyen un saludo del gerente general dando la bienvenida al nuevo colaborador. Estos suelen ser realizados por un proveedor externo a la empresa y, en la mayoría de los casos, no han sido actualizados en un par de años. También se usa diferentes videos para explicar procesos propios de las empresas o con información de soporte para algún taller o dinámica.

Además, se está incluyendo fotografías realizadas especialmente para la presentación de la inducción que incluyen a los propios colaboradores de la empresa. El uso creciente del material audiovisual es evidencia de que los públicos van cambiando y que es necesario dar o reforzar la información de manera más ágil.

Por otro lado, encontramos que, si bien se sigue entregando papeles, folletos y cierto material como manuales impresos, la mayoría cuenta con una versión digital que puede ser revisada mediante una carpeta compartida en el sistema de toda la organización o, en algunos pocos casos, en intranet o transmitidos por correo. Además, varias empresas han optado por entregar esta información necesaria y obligatoria mediante un dispositivo de almacenamiento masivo, ya sea un USB o CD, por lo general. Si bien no les asegura que sea leído, es considerado beneficioso, no solo por el cuidado del medioambiente, sino por

\footnotetext{
${ }^{8}$ Comunicación personal con Erika Claros (jefe de Selección y Gestión de Personal en IQ Farma), el día 24 de junio de 2015.
} 
ahorro económico en impresiones y tiempo de consulta del personal, además de la capacidad de almacenamiento y la facilidad de tenerlo listo para ser entregado por ocupar poco espacio físico.

Respecto a este último punto, encontramos un pequeño conflicto específicamente en la entrega del reglamento interno y, en algunos casos, del reglamento de seguridad y salud en el trabajo. Estos también están comenzando a ser entregados en dispositivos de almacenamiento masivo; sin embargo, se encontró cierto reparo a ello por parte de algunos líderes: "Una idea que tuvimos fue tener un USB para entregar las políticas, manuales y el reglamento interno, pero nos dijeron que no porque tienen la necesidad de que se firme un cargo de que lo recibieron",

El argumento de la necesidad del cargo firmado se escuchó en tres empresas más. No decimos que sea el único motivo por el cual no se entregue de manera digital; pero, al ser repetitivo, llamó la atención. Efectivamente, deben ser entregados por ley y dejar un cargo de recepción. En el caso del reglamento interno de trabajo, la norma dice: "El empleador está obligado a hacer entrega a los trabajadores de un ejemplar del Reglamento Interno de Trabajo o su modificación, presentado a la Autoridad competente, dentro de los cinco (5) días naturales de producido el referido acto" (Ministerio de Trabajo 2013), no especificando el medio ni la manera de entrega; y en el caso del reglamento interno de seguridad y salud en el trabajo, la ley estipula lo siguiente: "el artículo $75^{\circ}$ del Reglamento de la Ley de Seguridad y Salud en el Trabajo ha establecido la obligación de su entrega a todos los trabajadores, mediante medio físico o digital y bajo cargo" (Ministerio de Trabajo 2013). Es decir que el argumento presentado anteriormente no es válido. Otras empresas, para asegurarse, piden que abran el archivo y que luego firmen el cargo; también se ve casos, como en Linio y Procetradi, que lo manejan íntegramente de manera virtual y tienen como cargo un correo electrónico que avale la recepción.

Otro hallazgo, que si bien no logra alcanzar la tercera parte de las empresas investigadas, pero que es de gran relevancia mencionar, es la implementación del blending learning o b-learning; es decir, un modelo de aprendizaje virtual-presencial donde existe

\footnotetext{
${ }^{9}$ Comunicación personal con Lucía Rojas (practicante profesional en Hudbay), el día 17 de junio de 2015.
} 
una parte de la inducción que se brinda a manera de curso mediante alguna plataforma. Por ejemplo, DHL brinda el primer día de ingreso un USB que contiene pequeños cursos sobre la corporación que los nuevos trabajadores pueden revisar durante las tres primeras semanas antes de tener una evaluación y manteniendo aún una parte presencial. Otra empresa que optó por esta modalidad este año es Entel que, debido a problemas de coordinación y puntualidad con los facilitadores de distintas áreas, decidieron enfocar la inducción presencial a conocer la empresa y su cultura, colocando toda información complementaria y de las áreas en un curso virtual.

Un caso diferente se registró en la empresa Pacífico Seguros, ya que llegó a ser $b$ learning al integrar a su inducción la parte presencial. Con ello podemos ver que, a pesar de que ciertas tendencias nos lleven al uso de nuevas tecnologías, no se debe descuidar la parte humana:

[...] ahora tenemos muy interiorizado el tema de Vive Pacífico, que es tanto para nuestros clientes como para nuestros colaboradores, esto implica vivir seguro, con paz, tranquilo, sabiendo que alguien se está preocupando por ti. Entonces la idea es que vea esta filosofía desde que ingresa, y en verdad como te comentaba lo habíamos descuidado un poco porque solo recibía la inducción virtual, llenaba los papeles y se iba, ahora es b-learning ${ }^{10}$.

\subsection{Prácticas}

Las necesidades y formas de comunicación aumentan y se diversifican... simultáneamente, se desarrollan instrumentos, procesos, disciplinas y estrategias, de comunicación absolutamente inéditos. Tan inéditos como lo son las nuevas situaciones y las necesidades que acosan a las empresas en este horizonte del siglo XXI. (Costa 2000: 25)

\footnotetext{
${ }^{10}$ Comunicación personal con Silvana Fernández (subgerente de Desarrollo Organizacional en Pacífico Seguros), el día 9 de junio de 2015.
} 
Durante el trabajo de campo, se pudo reconocer la presencia de prácticas que forman parte del proceso de inducción. Estas son un complemento o refuerzo de la información brindada sobre la compañía, condiciones de trabajo y funciones. Sin embargo lo particular en ellas, o en algunas que describiremos, es como logran evidenciar la cultura de la organización y comunicar estratégicamente lo que necesitan de una manera no convencional pero cumpliendo con objetivos muchas veces elaborados con la intención de fidelizar e innovar.

Las prácticas más conocidas y replicadas por casi la totalidad de las empresas son el recorrido de las instalaciones, para que el nuevo trabajador pueda ubicarse en el espacio. En esta hay variantes como que lo haga acompañado por el jefe directo o por un personal de Recursos Humanos y que se rija a enseñarle las instalaciones o incluya la presentación del personal; así como obsequiar un kit de bienvenida que consta de merchandising propio de la empresa con el fin de que se vayan familiarizando con la marca. También varías empresas solían incluir el testimonio de un colaborador antiguo para que cuente su experiencia e historia de crecimiento.

Una práctica en particular que se ha visto en la tercera parte de las organizaciones entrevistadas y que está en proyecto en un par más, es el mentoring o un programa de tutor, que consiste en asignar a un compañero de trabajo usualmente un par de funciones para que sea su guía en cuestiones que no necesariamente se pueden resolver en las charlas de inducción, como dudas sobre el estilo de trabajo, cuestiones de comportamiento, cultura, etc.

Sin embargo, son muy pocas las empresas que tienen el programa realmente estructurado y menos las que capacitan a las personas que realizan este labor; pero también se pudo observar buenos ejemplos: la Clínica Internacional tiene un programa llamado "Formador de formadores" diseñado exclusivamente para capacitar a los tutores de los nuevos ingresos; Ban Bif tiene bien estructurada la práctica y el perfil de estos "padrinos" (como los llaman), que son personas con cinco años o más dentro de la empresa y que son considerados "embajadores de la cultura" del banco, con los cuales se genera un almuerzo de camaradería pagado por la empresa para iniciar una relación de confianza que permitirá 
al nuevo trabajador acudir a él para resolver dudas sobre el trabajo y hasta tradiciones de la empresa.

Otra práctica que está dándose en gran medida es la comunicación formal de los nuevos ingresos a toda la compañía, en especial si tienen diferentes sedes. Esta se suele dar por correo electrónico o intranet en empresas como Linio, Avianca, Intéligo y Natura, quienes suelen enviar un correo masivo donde se le da la bienvenida al nuevo ingreso incluyendo su foto y datos, así como sus pasatiempos, intereses, etc., haciéndolo más amigable sin incluir aspectos estrictamente profesionales. Sin embargo, si bien es una buena iniciativa, se encontró un par de casos donde, por falta de estrategia, no se tenía el impacto deseado:

[...] lo que se hacía era mandar un correo con la foto el nombre y el área a la cual estaba ingresando y funcionaba bien, la gente lo veía, pero dijeron que ya no, que todo sea mejor por intranet, y por eso medio es más frío, la gente solo se entera si entra y la verdad es que no es muy usada, entonces ahí sí estoy en discordancia con la gerencia, no soy una experta en comunicaciones pero diría que cada empresa tiene una manera de comunicarse, y no podemos forzar la comunicación o un método de comunicación para todos, y eso es justo lo que se quiere aquí, logar que la intranet sea la herramienta más usada solo porque es una plataforma que ha funcionado en otras empresas ${ }^{11}$.

Existe otro tipo de prácticas que empiezan a tomar más protagonismo y que, si bien la mayoría de empresas aún no los adopta, es importante para este estudio mencionarlas. Por un lado, tenemos las que llamaremos "prácticas lúdicas", que consisten en incluir talleres de integración, dinámicas y hasta, en algunos casos, juegos a manera de retos, buscando integrar a los colaboradores para que se sientan en confianza y romper el esquema de la inducción a modo de solo recibir información. Empresas como Saga Falabella, Entel, Chillis, entre otras, tienen un espacio para talleres, juego de roles, etc., que tienen como fin explicar de manera lúdica aspectos de las empresas, como los constantes cambios en el negocio, el trabajo en equipo, etc.

\footnotetext{
${ }^{11}$ Comunicación personal con Ornella Lambrusccini (jefa de Gestión Humana en Cecosami), el día 18 de junio de 2015 .
} 
Otras empresas, como IQ Farma, tienen concursos con premios para evaluar que hayan estado atentos y otras más innovadoras tienen prácticas como el rally, actividad lúdica de un banco donde al nuevo colaborador se le entrega una hoja con diez preguntas (como cuál es el pasatiempo del gerente de marketing, quién es tal persona y qué puesto tiene), que busca que los nuevos ingresos rompan con la timidez y generen camaradería y que, al terminar, hayan conocido diferentes áreas y un poco de la actitud del personal, quienes ya conocen esa práctica y están dispuesto a ayudar.

Por otro, se encontró un grupo de empresas que tienen institucionalizada una serie de prácticas que llamaremos emocionales, que involucran al colaborador más allá de lo laboral. Por ejemplo, Alicorp envía una maleta llena de productos de la empresa a la casa del nuevo colaborador usualmente quince días antes su ingreso, con el objetivo de darle la bienvenida, involucrar a sus seres queridos y mostrarles, al ver los productos, que ellos ya formaban parte de su vida antes de que ingrese a la empresa. Este tipo de prácticas inclusive salieron en anécdotas que contaban los entrevistados sobre antiguos empleadores, como aerolíneas o empresas grandes de cosméticos, que también les llevaron kits de bienvenida a sus casas y que fue muy valorado no solo por ellos, sino también por sus familias.

En Entel, por su parte, como dinámica, el primer día les hacen escribir lo que quieren lograr en la empresa, sus objetivos, lo que están dispuestos a dar; cuando cumplen tres meses, dejan ese papel en sus escritorios. También tienen otras más emotivas, como lanzar globos de helio con sus sueños y objetivos; lo cual, nos comentaba la entrevistada, tenía un impacto positivo no solo en los ingresantes, sino también con el público que veía la práctica al pasar por la puerta de su edificio y que alguna vez habían encontrado fotos de la práctica en redes sociales de personas externas. Otras empresas decoran sitios, organizan una bienvenida con detalles, banners, tarjetas con mensajes alentadores firmadas por toda el área y hasta financian almuerzos para que se puedan conocer extralaboralmente con sus jefes directos.

Además, se vio que algunas de las prácticas emocionales más elaboradas responden a empresas que tienen una cultura y filosofía bastantes sólidas y, en algunos casos, esta viene desde una matriz o al ser parte de una corporación, como en el caso de la 
empresa Intéligo que, al ser del grupo Intercorp, tiene una inducción más orientada a la persona, a acogerlo y darle una bienvenida cálida; por ello, la persona encargada nos comentaba que cuidan mucho los detalles y tenían varias prácticas que inclusive generaban feed back sin solicitarlo:

Y al final del día para incluir el tema familia que es digamos uno de los targets de Intéligo, les enviamos a su casa su postre favorito al final de la tarde pero a nombre de sus papás o su respectivo esposo e hijos, cosa que él llega y ve que ha recibido el presente, que su familia está contenta... es un nexo que queremos crear... el tema es anunciar a la familia que estamos contentos de que el colaborador nuevo ha ingresado y que existe un cariño, es todo el tema de feeling para con él y de que vamos a cuidarlo ${ }^{12}$.

Inclusive, otras empresas entrevistadas de la misma corporación, incluían prácticas con el mismo objetivo, como mandar una carta firmada por el gerente general dirigida a la familia donde se les daba la bienvenida a la empresa empleadora. Mencionaba que estaban felices por el ingreso, que debían sentirse orgullosos del nuevo colaborador y mostrarle su apoyo.

Finalmente, se encontró algunas prácticas que llamaré "no institucionalizadas", que nacen únicamente de la iniciativa de los nuevos compañeros de trabajo y suelen darse porque el área encargada no realiza nada más allá de instruir en conceptos básicos de la empresa y buscan dar una bienvenida más cálida: “Algunas áreas suelen tener un almuerzo de bienvenida... usualmente se da con el jefe y las personas que trabajan directamente con él. No lo financia la empresa, sale del bolsillo del jefe o de chanchita de todos los asistentes al almuerzo menos del nuevo" ${ }^{\prime 13}$.

Sin embargo, también se puede presentar por temas de cultura de la empresa que sus colaboradores tienen interiorizados:

[...] esto más es un rito que ya viene de nuestra cultura, nosotros decoramos el sitio en el que se va a ubicar el nuevo ingreso con globos, serpentinas, y cada área hace una

\footnotetext{
${ }^{12}$ Comunicación personal con Lizeth Orcón (analista senior de Gestión y Desarrollo Humano en Intéligo SAB), el día 23 de junio de 2015.

${ }^{13}$ Comunicación personal con Agatha Hau (analista de sistemas de Gestión de Seguridad y Salud en el Trabajo), el día 2 de mayo de 2015.
} 
tarjeta después de investigar que gustos tiene ese colaborador con su $\mathrm{CV}$ o en Facebook y se le arma algo visual con algunos dibujos que firma todo el área deseándole éxitos y buenos deseos ${ }^{14}$.

\subsection{Tendencias}

En esta parte, se resaltará dos aspectos que se han convertido en un detalle importante de la inducción: primero, la tendencia actual a la digitalización de contenidos; luego, incluir la cultura corporativa como parte importante y hasta fundamental del proceso investigado.

\subsubsection{Acceso a la información y la tendencia a la digitalización del contenido}

Como vimos en la parte de medios, se comienza a evitar el papel para pasar a dispositivos de almacenamiento masivo; pero, no solo eso, sino que los mismos encargados del proceso reconocen que la información brindada es de gran cantidad, por lo que están optando por hacer resúmenes, mostrar lo esencial dentro de las presentaciones y tener un espacio (no necesariamente una intranet) donde los colaboradores pueden entrar y revisar la información.

Algunas empresas, como Deloitte, se adelantaban a las posibles preguntas sobre lo visto en la inducción o temas que no se tocaron tan afondo, enviando un correo al finalizar el día de la charla, donde podían revisar la información vista en la inducción, además de los manuales, reglamentos y números y correos del personal clave. Este tipo de acciones agilizan el proceso de terminar con la incertidumbre y desconocimiento con el que ingresan todos.

${ }^{14}$ Comunicación personal con Lorena García (especialista corporativo en Selección en Casa Andina), el día 25 de junio de 2015. 
Si bien son pocas aun las empresas que tienen un espacio en alguna plataforma virtual para almacenar contenido o para dar una especia de curso b-learning, están bastante inmersas y con objetivos claros respecto a manejar contenido e información de manera virtual/digital, inclusive buscando un impacto con la inducción no solo en el nuevo colaborador:

Aparte de estar en medio del proyecto de tener nuestro propio espacio en la intranet, estamos en coordinaciones con el área de tecnología y de selección, queremos potenciar lo que es el Linkedin, y subir fotos de los grupos y poner y enseñar cómo es la inducción en el BBVA, y queremos darle más impulso, queremos que se vea en redes, eso mueve ${ }^{15}$.

\subsubsection{La cultura expresada desde la inducción}

Cuando nuevas personas ingresan a una organización, deben saber en hacia dónde dirigir sus actividades y esfuerzos, sin embargo - Según Chiavenato (2009)- de nada sirve tratar de orientar a las personas dentro de la organización si ésta no sabe hacia dónde quiere ir, ya que solo así las personas podrán ayudar en su trayectoria y desarrollo, además, señala que si bien conocer la misión y la visión de la organización es fundamental, lo más importante para ese fin es adaptarse a su cultura organizacional

En el caso de las empresas que formaron parte de la investigación, la mitad tiene o ha incorporado recientemente un espacio especial para hablar sobre la cultura de su organización, no solo mencionando la misión, visión y valores en un ppt, sino también haciendo talleres para interiorizarlos, en donde hablan sobre sus ritos, las características de los colaboradores de la empresa, etc.

Las empresas que cuentan con este espacio, la consideran vital principalmente por que los nuevos ingresos son personas que tienen actitudes, modos de trabajar, entre otros que, de no poder encajar con los de la empresa, podrían terminar en una mala adaptación o una deserción:

\footnotetext{
${ }^{15}$ Comunicación personal con Paola Carozzo (subgerente de Áreas de Apoyo, Recursos Humanos, en BBVA), el día 19 de mayo de 2015.
} 
El tema de la cultura es fundamental porque te permite abrir los ojos y ver que estás entrando a una empresa donde se valoran cosas que pueden ser muy símiles a ti o muy diferentes a ti, entonces si tú eres una persona que comparte los valores y tienes la misma filosofía que es recontra humana vas a decir que chévere trabajar acá [...] y si eres una persona que no comparte los valores es una apertura de ojos para darte cuenta de que esta no es una empresa para ti y que te tienes que ir [...] la empresa no va a estar cambiando por ti y viceversa, será solo una relación disfuncional que va a llevar al fracaso ${ }^{16}$.

Cabe resaltar que existe una relación bastante interesante y es que son justo las empresas que cuentan con un apoyo expreso de los altos mandos de la organización hacia el proceso de inducción, ya sea con su participación activa en esta o con la autorización de presupuesto y transmisión de su importancia, son las que tienen una estructura más definida del proceso y una cultura sólida que transmitir; por ende, son la mayoría que forman parte de la tendencia. Sin embargo, existen también las empresas que, si bien no tienen un apoyo o por otro lado no tienen el presupuesto para realizar algo más elaborado, consideran que tocar el tema de la cultura es fundamental por un tema más de identificación. Por ejemplo, la empresa Grupo Siglo, que brinda servicios de auditoría y contabilidad tercerizada, lo considera vital debido a que sus colaboradores pasan más tiempo en las oficinas de los clientes que en las suyas, sintiéndose a veces más identificados con ellos y hasta adaptando su cultura.

\subsubsection{Proyectos a implementar}

Finalmente, las empresas consideran que, como todo proceso, siempre puede mejorarse y están incluyendo en sus planes a mediano y largo plazo proyectos de mejora para la inducción en tres aspectos que mencionaré según la cantidad de empresas que los tienen.

En primer lugar, casi la mitad está buscando estructurar mejor el proceso y su contenido actual, queriendo también darle mayor importancia al seguimiento del impacto

${ }^{16}$ Comunicación personal con Antonio Maurer (jefe de Cultura Organizacional en Mineras Chinalco Perú SA), el día 18 de junio de 2015. 
de la inducción a los nuevos ingresos, así como a la inducción al puesto, que por lo general depende del jefe inmediato.

Seguidamente, buscan también innovar y/o incluir ciertas prácticas y detalles de bienvenida que hagan más cálida la inducción y, ya en menor cantidad, hay empresas que están en pleno proceso de adquirir una plataforma digital para poder incluir en ella parte de la inducción o, por otro lado, insertar — como en el caso de la Universidad Simón Bolívar - una pestaña especial dentro de su nueva intranet exclusiva para que tanto nuevos como antiguos colaboradores puedan acceder a la información que se brindó el día de la inducción.
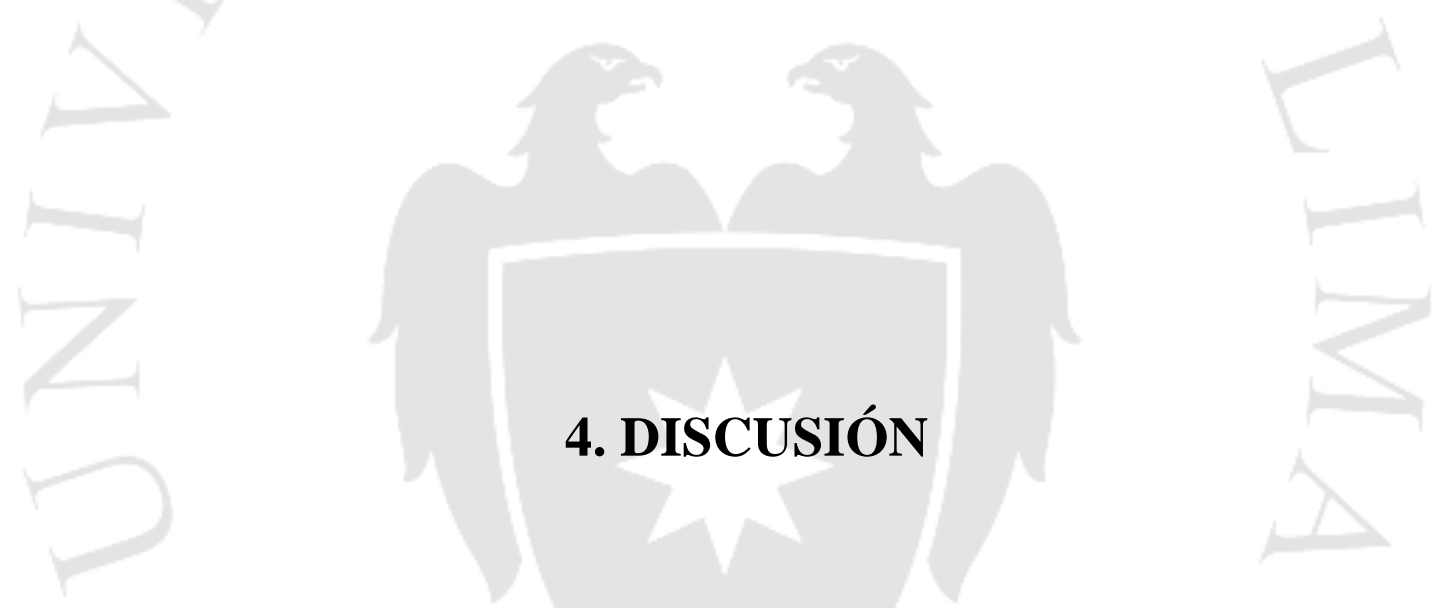

Cuando se inició la investigación, se tenía como objetivo evaluar el componente comunicativo en el proceso de inducción al personal, esto sobre la base de los medios utilizados durante el proceso, sus respectivos contenidos, además de las prácticas que podrían formar parte del mencionado proceso.

Al ir adentrándonos en el trabajo de campo, se logró conocer ambos indicadores. En el caso de los medios, se vio materiales estandarizados para las empresas, así como una tendencia a elaborar presentaciones de corte más audiovisual, saliendo de lo tradicional, incluyendo también el uso de diferentes unidades de almacenamiento y acceso a la información. En cuanto a las prácticas, si bien no fueron encontradas en la mayoría de las empresas, se vio que no solo fueron implementadas para ir desarrollando ciertas aptitudes o 
habilidades, sino que hasta llegaban a involucrar emocionalmente a los nuevos colaboradores; ambos hallazgos se estaban desarrollando en las organizaciones recién de manera institucionalizada este último año o máximo hace dos, debido a que se han dado cuenta que hasta en los procesos manejados de manera interna es importante adaptarse a los cambios que exige el mundo laboral.

Además de conocer las características, importancia e impacto de los dos aspectos anteriormente mencionados (medios, contenidos y prácticas), se encontraron otros hallazgos relevantes que en cierta forma se desprenden o relacionan directamente con ellos.

Por un lado, se vio como tendencia la digitalización de contenidos. Los medios comenzaban a dejar de ser exclusivamente físicos como manuales y guías impresas; se comienza a tener acceso a la información mediante intranet, links, direcciones compartidas $\mathrm{y}$, en algunos casos de minorías, se complementaban con una parte de la inducción de manera virtual en distintas plataformas. Por otro lado, algunas empresas y con posibilidad de ser más, mencionaron que estaba dentro de sus planes a mediano plazo incluir un espacio destinado a hablar e introducir a los nuevos en la cultura de la organización. Si bien esta cultura puede transmitirse de manera oral, la refuerzan o sin querer la evidencian con las prácticas realizadas, ya que estas manifiestan las características y la orientación e importancia que le dan las diferentes empresas a ciertos temas como, por ejemplo, lograr una identificación del nuevo ingreso con la compañía.

Es importante mencionar que el proceso de inducción es fundamental en la incorporación de nuevos colaboradores, como bien menciona Dessler (2009). Este debería cumplir con ciertos objetivos, como sentirse bienvenido, adquirir un conocimiento general sobre la organización y su trabajo, así como empezar el proceso de conocer la manera en que la empresa actúa y hace las cosas. Por todo ello, es necesario que se dé al iniciar las labores; sin embargo, la realidad, al menos en las empresas peruanas, es que no se da siempre en los primeros días.

Hablando también de los objetivos mencionados, se puede observar que casi la totalidad de las empresas logran dar información general de la empresa y del puesto; sin embargo, son el dar una bienvenida cálida y lograr que comiencen a conocer el actuar o cultura de la empresa, lo que no se ve en todas las organizaciones, pero se nota una 
creciente preocupación por implementar charlas o prácticas que permitan alcanzar esos objetivos.

Además — como menciona Jaime Grados (2003)—, este proceso, más que un gasto, es una inversión; ya que impacta en la motivación y, por ende, en el rendimiento de los nuevos ingresos. Esto se pudo validar dentro de la investigación ya que muchos de los entrevistados consideraban que era una inversión que inclusive a la larga podría ser considerada de una estrategia de retención.

También se encontró importante la participación de los líderes de la organización, de quienes dependen las facilidades para su desarrollo, como tiempo y presupuesto; incluso, coincidía que las empresas que tienen este proceso mejor estructurado y con más componentes tenían implicados a sus líderes. Se recomienda su participación activa, ya que ellos son el ejemplo y podrían servir hasta de inspiración y guía para los nuevos trabajadores, dando también la imagen de que la empresa en todos sus niveles se preocupa por ellos (Pincai y Rentería 2014).

Este artículo es de mucha importancia, ya que revela que la inducción al personal es realmente relevante en el proceso de inserción y adaptación del nuevo colaborador; además, basándonos en las treinta y tres realidades estudiadas, se puede afirmar que las empresas privadas lo reconocen e incluso han comenzado a darle un nuevo enfoque dentro de sus posibilidades y orientación para conseguir colaboradores más comprometidos e informados.

Se evidencia también que aún falta mucho por hacer respecto al tratamiento de la información brindada durante el proceso. La cantidad de información que reciben es abundante y, si bien una persona suele compilar las información y darle un solo formato, son pocas las empresas que se toman el trabajo de revisar la coherencia con el perfil de la empresa, que no se duplique información, que reciban un contenido estratégicamente diseñado para ese público que se encuentra lleno de dudas y expectativas.

Sin dejar de mencionar la capacidad de algunas y resistencia de otras para adaptarse a los cambios, con las nuevas tecnologías se puede lograr presentaciones más eficientes, así como la simplificación de contenidos y acceso a la información; además de 
las nuevas tendencias de intentar fidelizar, incluyendo talleres, dinámicas y prácticas, generando un vínculo más humano, involucrando desde el inicio al nuevo ingreso con la empresa y sus objetivos.

Durante la realización del presente artículo, se encontraron también ciertas dificultades. Primero, existe un vacío en el campo de la investigación respecto al componente comunicativo en el proceso de inducción: no dan a conocer la manera ni medios que deberían o se usan para llevar este a cabo, sin mencionar que no se habla del tratamiento de información y su consolidación. Tampoco se encontró información de las prácticas como tal. Por otro lado, también se debe mencionar la negación de algunas empresas a conceder la entrevista debido a falta de tiempo del público objetivo y, en algunos casos, por políticas de confidencialidad.

Finalmente, cabe mencionar, para las siguientes investigaciones a realizar, la necesidad de tomar en cuenta la estructura del seguimiento que se le hace a la inducción, ya que podría convertirse en un punto clave. Por otro lado, se debería incluir el punto de vista de los colaboradores respecto a la importancia e impacto de este proceso, sobre la información, los medios y plataformas mediante los cuales les gustaría recibirla. Si bien se consiguió aproximaciones de los entrevistados al contar sus experiencias al recibir inducción en anteriores y actuales empleos, considero que sería una perspectiva que complementaría los resultados de esta investigación. 


\section{REFERENCIAS}

Arellano Reinoso, Vanessa Tatiana (2014). "Implementación de un plan de inducción y re inducción tendiente a que permita que el personal se identifique con los objetivos organizacionales". Tesis de Licenciatura. Universidad Central de Ecuador. <http://www.dspace.uce.edu.ec/bitstream/25000/3647/1/T-UCE-0007-148.pdf>.

Calvo Pérez, Javier (2012) La Oreja en el piso. Una cultura para el ejercicio del liderazgo. Perú: Santillana. S.A.

Cárdenas, Alejandro et al. (2009). "El programa de bienvenida. Guía de implantación: costes, herramientas y seguimiento". Wolters Kluwer. Año 22, núm. 233. Capital Humano.

Chiavenato, Idalverto (2009). Gestión del Talento Humano. México: Mc Graw Hill Educación.

Chiavenato, Idalverto (2011). Administración de recursos humanos. El capital humano de las organizaciones. México: Mc Graw Hill Educación.

Costa, Joan y López, Mariana (2000). El poder de la comunicación institucional. Buenos Aires: Revista Imagen S.A.

Dessler, Gary (2009). Administración de recursos humanos. 11. a edición. México: Pearson Educación.

García, Carlos (1999). "Estudio sobre estrategias de inserción profesional en Europa". $\begin{array}{lllll}\text { Revista Iberoamericana de Educación. } & \text { Núm. }\end{array}$ <http://www.rieoei.org/oeivirt/rie19a03.htm>.

García, Claudia y Oliveiro, Yajaira (2014). "Programa de inducción para los servidores públicos del Centro de Servicios Chacao de la Corporación Eléctrica Nacional”. Tesis de Licenciatura. Universidad Nueva Esparta (Caracas). <http://miunespace.une.edu.ve/jspui/bitstream/123456789/2423/1/TGC0015.pdf>.

Gómez, Luis; Balakin, David y Cardy, Robert (2008). Gestión de recursos humanos. 5. edición. Madrid: Pearson Educación. 
Grados, Jaime (2003). Reclutamiento, selección, contratación e inducción del personal. 3. ${ }^{a}$ edición. México: El Manual Moderno.

Huerta, Rocío (1991). "La comunicación organizacional, los programas de inducción y su importancia en la dinámica de la organización”. Tesis de Licenciatura. Universidad de Lima.

Jaramillo, Emiliano y Díaz, Carlos (2011). "Pertinencia y contribución del proceso de inducción en el desempeño de los funcionarios vinculados al banco agrario de Colombia regional cafetera. Estudio de caso". Tesis de Maestría. Universidad Autónoma de

Manizales. <http://repositorio.autonoma.edu.co/jspui/bitstream/11182/314/1/PERTINENCIA\% 20Y\%20CONTRIBUCION\%20DEL\%20PROCESO $\% 20$ DE\%20INDUCCION $\% 20$ EN\%20EL\%20DESEMPE\%C3\%910\%20DE\%20LOS\%20FUNCIONARIOS\%20 VINCULADO.pdf $>$.

Jofre, Roxana (2007). "Programas de inducción de personal". <http://www.gestiopolis.com/programas-de-induccion-de-personal/>.

Laos, Nancy et. al (2013). Compendio de normas sobre legislación laboral del régimen privado. <http://www.mintra.gob.pe/archivos/file/SNIL/compendio_normas/COMPENDIO_ NORMAS_LABORALES_MINTRA_2013.pdf $>$.

Mejía, Julio (2000) "El muestreo en la investigación cualitativa". Investigaciones sociales. Año IV, núm. 5, pp. 165-180.

Mendoza Jiménez, Tania (2013). "Elaboración de un manual de inducción para la imprenta Don Bosco". Universidad Politécnica Salesiana. Tesis de Licenciatura. 〈http://dspace.ups.edu.ec/bitstream/123456789/5618/1/UPS-CT002792.pdf〉.

Ministerio de Trabajo (2013). "Anexo 2. Modelo de reglamento interno de seguridad y salud en A FT el trabajo". <http://www.mintra.gob.pe/archivos/file/CNSST/anexo2_rm050-2013.pdf>.

Paillacho Anchapaxi, Nancy Isabel (2011). "El diseño y aplicación de un manual de inducción para mejora del clima organizacional del personal que labora en el Museo de la Ciudad de Quito". Tesis de Licenciatura. Universidad Central de Ecuador. <http://www.dspace.uce.edu.ec/bitstream/25000/1741/1/T-UCE-0007-42.pdf〉. 
Pincai, Rebeca y Rentería, Lita (2014). "Diseño de un manual de descripción de funciones y de un manual de inducción para el personal de la empresa Multimundo S.A.”. Tesis de Licenciatura. Universidad Católica de Santiago de Guayaquil. <http://repositorio.ucsg.edu.ec:8080/bitstream/123456789/1651/1/T-UCSG-PREFIL-CPO-4.pdf>.

Valera, Ricardo y Dessler, Gary (2011). Administración de recursos humanos. Enfoque latinoamericano. México: Pearson Educación.

Villafañe, Justo et al. (2014). La comunicación empresarial y la gestión de los intangibles en España y Latinoamérica. Barcelona: Gedisa S.A. 


\section{ANEXO}

Lista de empresas entrevistadas para la investigación

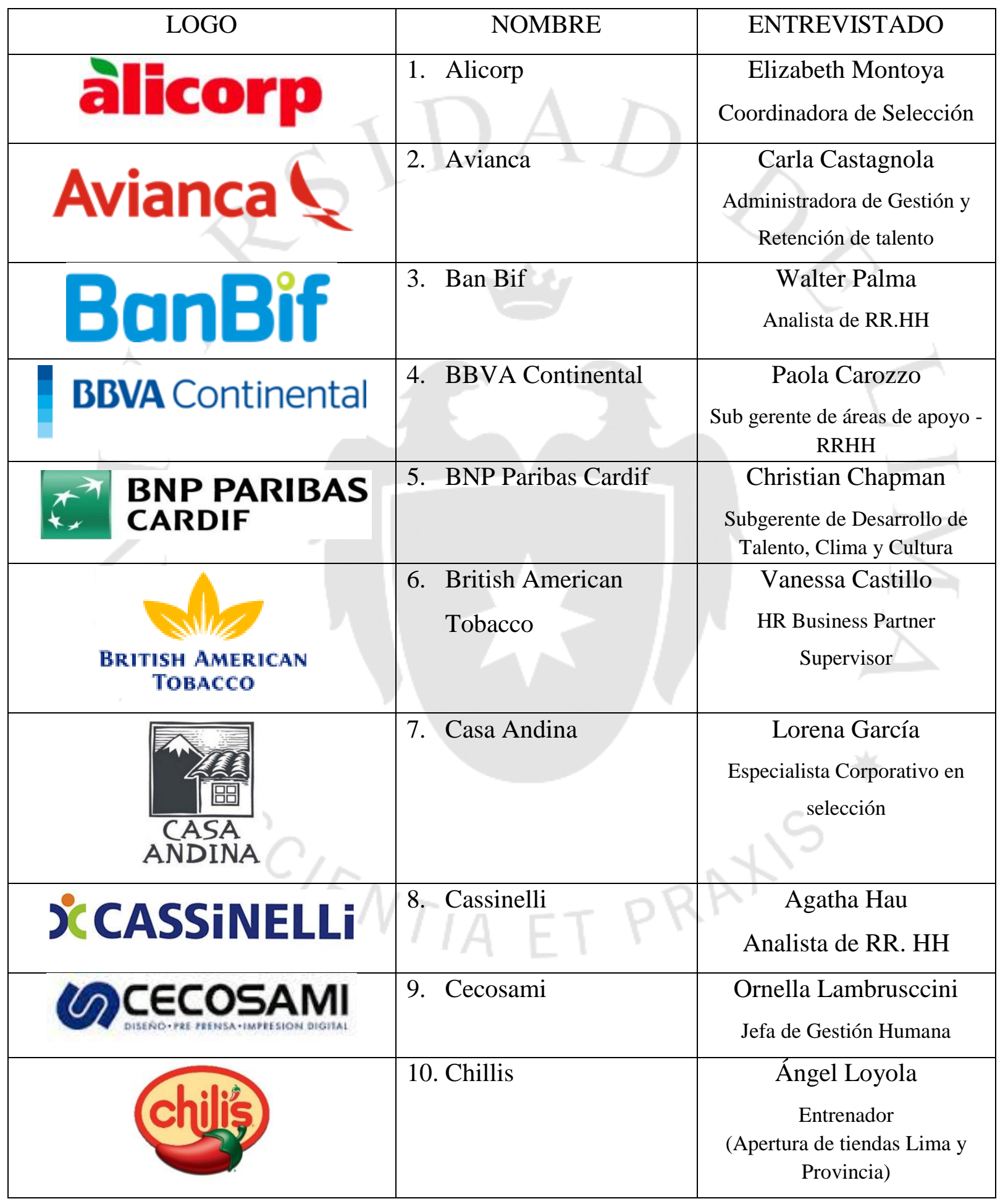




\begin{tabular}{|c|c|c|}
\hline & 11. Chinalco & $\begin{array}{l}\text { Augusto Maurer } \\
\text { Jefe de Cultura } \\
\text { Corporativa }\end{array}$ \\
\hline 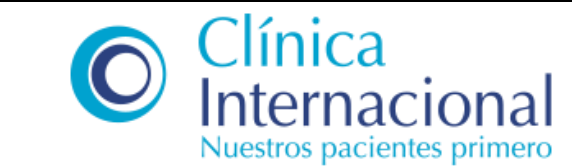 & 12. Clínica Internacional & $\begin{array}{c}\text { Percy Lenci } \\
\text { Coordinador de Gestión de } \\
\text { Talento }\end{array}$ \\
\hline & 13. Deloitte & $\begin{array}{c}\text { Brenda Balois } \\
\text { Asistente de Talento }\end{array}$ \\
\hline IDI & 14. Deprodeca & $\begin{array}{l}\text { Giuliana Velarde } \\
\text { Analista de RR.HH }\end{array}$ \\
\hline & 15. DHL & $\begin{array}{c}\text { Ángela De la Cruz } \\
\text { Practicante Profesional de } \\
\text { Desarrollo y capacitación }\end{array}$ \\
\hline & 16. Divemotor & $\begin{array}{c}\text { Klira Encinas } \\
\text { Jefe de Gestión Humana }\end{array}$ \\
\hline & 17. Entel & $\begin{array}{c}\text { Alejandra Kisic } \\
\text { Jefa de Recursos Humanos }\end{array}$ \\
\hline & 18. Grupo Siglo & $\begin{array}{l}\text { Milagros Angulo } \\
\text { Gerente de gestión de talento }\end{array}$ \\
\hline $\mathrm{c}$ & 19. Hayduk & $\begin{array}{c}\text { Angélica Gago } \\
\text { Jefa de Desarrollo Humano }\end{array}$ \\
\hline & 20. Hudbay & $\begin{array}{c}\text { Lucía Rojas } \\
\text { Practicante Profesional de } \\
\text { RRHH } \\
\end{array}$ \\
\hline acceso group & 21. Imedia & $\begin{array}{c}\text { David Carpio } \\
\text { Analista de Desarrollo } \\
\text { Organizacional }\end{array}$ \\
\hline IN SAB & 22. Intéligo SAB & $\begin{array}{c}\text { Lizeth Orcón } \\
\text { Analista Senior de GDH }\end{array}$ \\
\hline
\end{tabular}




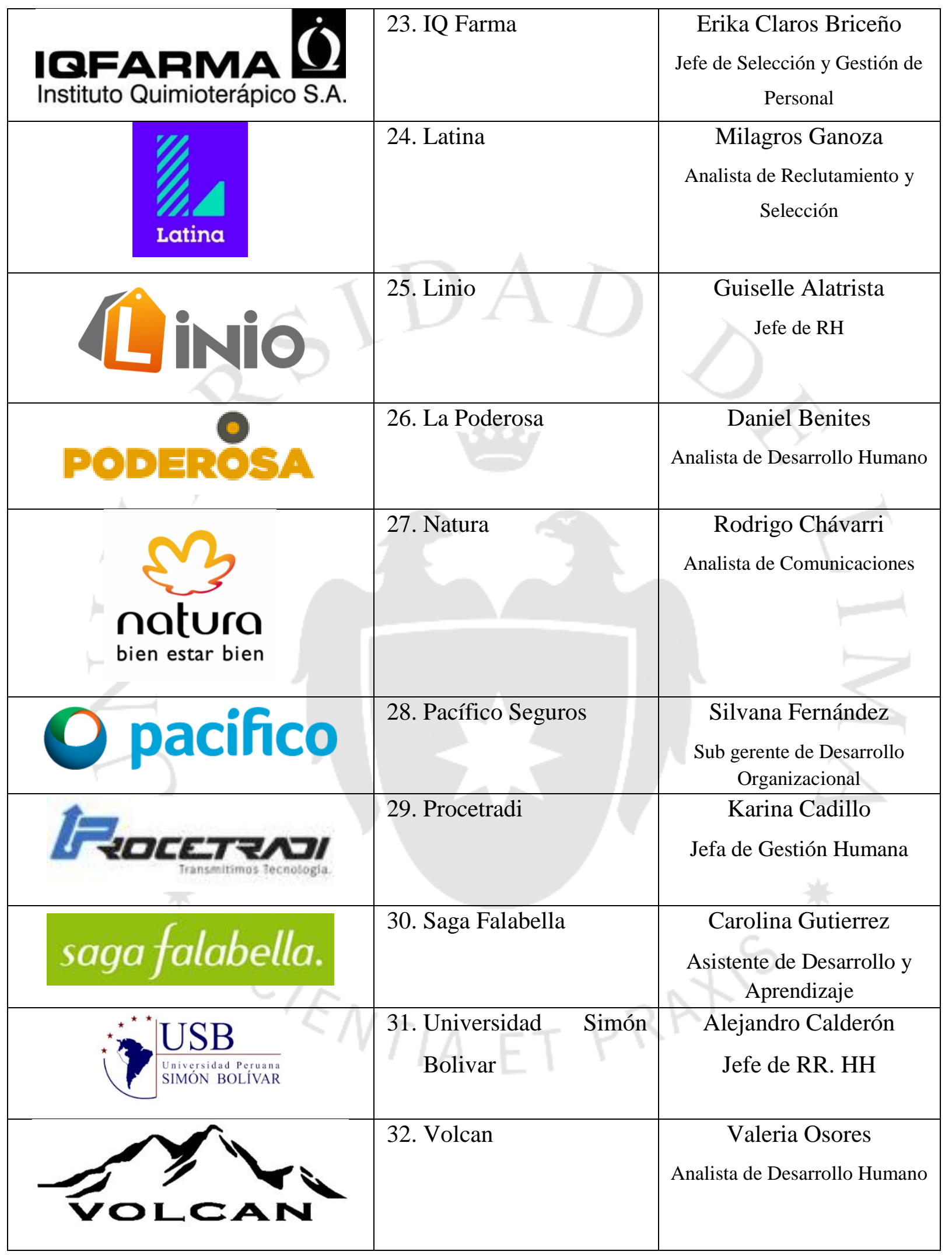

